\title{
PERFIL SOCIOCULTURAL DOS CANDIDATOS AOS CURSOS DA ÁREA DE ENGENHARIA NO VESTIBULAR DA UFF 2011
}

\author{
Geremias de Mattos Fontes Neto ${ }^{1}$ \\ José Rodrigo de Moraes ${ }^{2}$ \\ Luz Amanda Melgar Santander ${ }^{3}$ \\ Mônica de Aquino Galeano Massera da Hora ${ }^{4}$
}

\begin{abstract}
Resumo: Os cursos de Engenharia são de extrema importância para o país e para melhorar as condições de vida da população em geral, e exigem dos alunos bons conhecimentos em ciências exatas. A preocupação excessiva com o vestibular e a precocidade da escolha da área profissional pode levar a evasão nos cursos de graduação em Engenharia ou o prolongamento do tempo de formação do aluno. Utilizando os dados do Vestibular da UFF 2011, este trabalho estabeleceu a associação entre características demográficas, socioeconômicas e culturais e a escolha dos candidatos pelas áreas de Engenharia, relativamente às demais áreas de Ciências Exatas, e as áreas de Ciências Humanas/Biológicas. Entre os resultados do trabalho, destaca-se a maior chance pela escolha das áreas de Engenharia entre os candidatos do sexo masculino, de 15 a 18 anos, com motivação financeira, filhos de pais mais escolarizados, com maior renda mensal familiar, que concluíram o ensino médio em 2009 ou 2010 e que apresentam domínio de alguma língua estrangeira. A complexidade da escolha profissional mostra a importância da orientação, desenvolvida por uma equipe multiprofissional em escolas do ensino médio, para auxiliar os alunos nas suas escolhas profissionais.
\end{abstract}

Palavras-chave: Escolha da área profissional, Vestibular da UFF, Cursos de Engenharia, Modelo logístico multinomial.

Abstract: Engineering courses are extremely important for Brazil and for improving the general population's living conditions. Engineering students are required to have good knowledge of physical sciences. Excessive concern regarding the entrance examination and choosing a professional field at an early age may lead students to drop out of undergraduate engineering courses or prolong the time spent in training. The present study used data from the entrance examination of Universidade Federal Fluminense in 2011, in order to establish correlations between candidates' demographic, socioeconomic and cultural characteristics and their choice of engineering in relation to other fields of the physical sciences and fields within human and biological sciences. Among the results from this study, it can be highlighted that the chance that engineering would be chosen was higher among the following candidates: males; ages 15 to 18 years; financially motivated individuals; children of better-educated parents; from families with higher monthly income; with high school concluded in 2009 or 2010; and with mastery of a foreign language. The complexity of choosing a professional shows the importance of guidance provided by multiprofessional teams in high schools, so as to aid students in their choice of profession.

\footnotetext{
Coordenação de Agropecuária, Instituto Brasileiro de Geografia e Estatística. E-mail: geremias.neto@ibge.gov.br

${ }_{2}^{2}$ Instituto de Matemática e Estatística, Universidade Federal Fluminense. E-mail: jrodrigo78@est.uff.br

${ }^{3}$ Instituto de Matemática e Estatística, Universidade Federal Fluminense. E-mail: luzamandams@yahoo.com.br

${ }^{4}$ Coordenação da Pós-Graduação em Defesa e Segurança Civil, Universidade Federal Fluminense. Email: dahora@vm.uff.br.
} 
Keywords: Choice of professional field, Entrance examination of Universidade Federal Fluminense, Engineering courses, Multinomial logistic model

\section{INTRODUÇÃO}

Os cursos de engenharias são de extrema importância para o desenvolvimento de um país e para a melhoria das condições de vida da população. Segundo Longo (1996), os engenheiros estão entre os principais profissionais que contribuem para $o$ desenvolvimento de um país, por serem responsáveis por muitas das inovações tecnológicas na sociedade. Este autor ressalta ainda que o crescimento mais elevado da economia brasileira pode ser atribuído diretamente a dois fatores: o maior número de profissionais especializados nas áreas de Engenharia e a demanda da população por educação superior. Este segundo fator deve-se ao fato da educação superior ser formadora de mão de obra altamente qualificada e por constituir importante meio para produção científico-tecnológica (Castanho \& Freitas, 2006). Além disso, o diploma universitário representa um símbolo de status socioeconômico, pois aumenta a chance de um indivíduo conseguir emprego num mercado de trabalho cada vez mais competitivo e de desfrutar de uma vida mais confortável.

Alguns acontecimentos recentes têm sido especialmente influentes para o aumento da demanda por cursos das áreas de Engenharia, como a instalação em andamento do Complexo Petroquímico do Estado do Rio de Janeiro (COMPERJ), localizado no município de Itaboraí, e ao aumento da produção de petróleo no Brasil (Cardoso et al., 2011), além da recente descoberta de grandes quantidades de petróleo na camada do Pré-Sal (Confea, 2010) e da construção de grande quantidade de empreendimentos imobiliários e investimentos na infraestrutura para realizar a Copa do Mundo de 2014, no Brasil, e as Olimpíadas de 2016, na cidade do Rio de Janeiro (Guimarães et al., 2011).

A preocupação excessiva com o vestibular pode sobrepor a atenção necessária à precocidade da escolha da carreira profissional de nível universitário (Sparta \& Gomes, 2005), o que pode trazer uma série de consequências para os alunos ingressantes em universidades. No caso das áreas de Engenharia, tais consequências estão relacionadas ao nível de desempenho dos alunos que, em geral, apresentam maior dificuldade nas disciplinas, sobretudo naquelas relativas aos períodos iniciais dos cursos, como cálculo e física.

Os alunos ingressantes nas áreas de Engenharias devem apresentar bons conhecimentos em matemática, física e química oriundos do ensino médio, que são disciplinas necessárias para a formação do aluno nestas áreas. No Brasil, o ensino médio é considerado uma etapa crucial na trajetória escolar dos jovens, uma vez que a conclusão do nível médio, além de aumentar a chance de inserção desses jovens no mercado de trabalho, torna possível a sua participação no vestibular e, uma vez aprovados, a sua entrada no nível superior de ensino. Segundo Sacavino (2006), embora tenha ocorrido uma expansão da oferta do ensino médio no Brasil, prioritariamente por meio da ampliação do ensino público, esta oferta não foi acompanhada pela qualidade necessária.

A falta de conhecimento sobre a área escolhida, aliada a falta de adaptação ao ambiente universitário, pode levar a evasão dos alunos, ou então, prolongar o tempo de formação no curso. Diante das exigências e requisitos necessários para a formação dos alunos nas áreas de Engenharia, é de extrema importância que o candidato goste da área escolhida e, também, que se dedique o maior tempo possível ao curso para assimilar os conteúdos das diferentes disciplinas, que façam cursos complementares de informática e de línguas estrangeiras.

Como apontado por Silva \& Cecílio (2007), os alunos, uma vez formados nas áreas de Engenharias, devem continuar se atualizando, para que não sejam excluídos do mercado de 
trabalho. Além de conhecimentos técnicos, os alunos das áreas de Engenharias devem se preocupar ainda com as questões políticas, econômicas e, sobretudo, com a responsabilidade social associada a sua futura formação profissional (Bianchini et al., 2011; Filho et al., 2011).

Este trabalho tem como objetivo analisar as características demográficas, socioeconômicas e culturais dos candidatos inscritos no Vestibular da UFF 2011, e identificar a associação entre essas características e a escolha pela área de engenharia, comparativamente às demais áreas de ciências exatas, e as áreas de ciências humanas ou biológicas.

\section{MATERIAL E MÉTODOS}

Para a realização deste trabalho serão utilizados os dados levantados no requerimento de inscrição e no questionário sociocultural do Vestibular da UFF 2011, ambos preenchidos pelos candidatos no momento da inscrição no concurso Vestibular UFF 2011, via internet, no período de 3 a 31 de agosto de 2010 (mas prorrogado até o dia 9 de setembro de 2010). O preenchimento do requerimento de inscrição é obrigatório no processo de inscrição, enquanto $o$ questionário sociocultural é facultativo.

O questionário sociocultural é composto por questões de âmbito social, econômico e cultural. São feitas perguntas referentes a educação do candidato e de seus pais, as escolhas do candidato quanto ao vestibular, a renda e a condição econômica da família, além de perguntas sobre preferências culturais e atividades exercidas pelo candidato. $\mathrm{O}$ requerimento de inscrição contém informações pessoais necessárias para comprovação da solicitação de inscrição no concurso Vestibular UFF 2011, além do curso desejado.

No estudo, considerou-se apenas os candidatos inscritos para os cursos disponíveis nos campi de Niterói. Além disso, os candidatos que não responderam pelo menos uma das informações de interesse foram excluídos do estudo.
A variável resposta do estudo foi a área escolhida pelo candidato, definida a partir da informação sobre o curso escolhido no requerimento de inscrição no Vestibular da UFF 2011. Deste modo, a variável "área escolhida" apresentou três categorias: 1) áreas de engenharia; 2) demais áreas de ciências exatas; 3) áreas de ciências humanas ou biológicas.

Para explicar a escolha da área, foram adotadas variáveis que se referem às características socioeconômicas e culturais levantadas no questionário sociocultural e às características demográficas contidas no requerimento de inscrição, à saber: Sexo (masculino, feminino), Faixa etária (15 a 18 anos; 19 a 20 anos; 21 anos ou mais), Cidade de residência (Rio de Janeiro; Niterói; São Gonçalo; Outra), Tipo de estabelecimento em que cursou o ensino médio (todo ou maior parte em escola pública; todo ou maior parte em escola particular),Ano de conclusão do curso de ensino médio (2010; 2009; 2008 ou antes), Curso frequentado (além do ensino médio) para prestar o Vestibular (não frequentou; frequentou Curso pré-vestibular e/ou curso de matérias/ isoladas), Quantidade de exames vestibulares prestados anteriormente(não prestou; um; dois ou mais), Motivo principal para a escolha do curso (adequação as aptidões pessoais; maior oferta no mercado de trabalho/recompensa financeira mais promissora; outro), Cor (branca, não branca), Nível de instrução de seu pai (no máximo fundamental; médio; superior ou pós-graduação), Nível de instrução de sua mãe (no máximo fundamental; médio; superior ou pós-graduação), Renda mensal familiar (até 3 salários mínimos; mais de 3 a 10 salários mínimos; mais de 10 salários mínimos), Situação de trabalho (não trabalha; trabalha), Local de moradia(na casa dos pais; outro) e Domínio de alguma língua estrangeira (sim; não).

Para análise das informações foi utilizado o software SPSS for Windows, versão 20.0, e ajustou-se o modelo de regressão logística multinomial para explicar a escolha da área pelos candidatos, considerando as suas 
características

socioeconômicas, demográficas e culturais. No ajuste do modelo, utilizou-se o método de máxima verossimilhança (Kutner et al., 2004; Marôco, 2010).

A partir do modelo selecionado, foram obtidas medidas de razão de chances, que são medidas de associação utilizadas para avaliar o sentido e o grau da associação entre as características dos candidatos e a chance de escolherem as áreas de Engenharia, relativamente as áreas de Ciências Exatas e as áreas de Ciências Humanas/Biológicas. Para avaliar a significância das associações, utilizou-se $o$ teste de razão de verossimilhanças e o teste de Wald, considerando o nível de significância de $5 \%$.

\section{RESULTADOS E DISCUSSÃO}

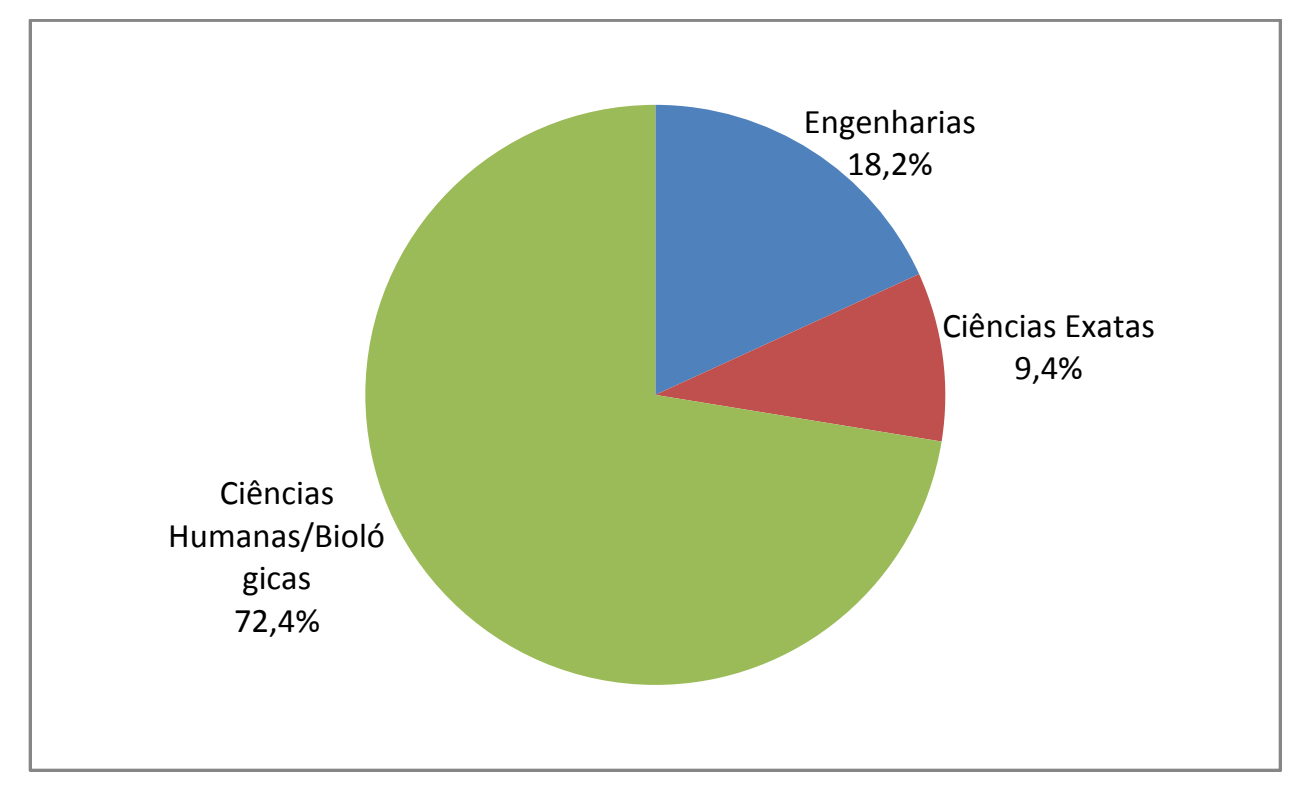

Figura 1: Distribuição de candidatos por área escolhida 
Tabela 1: Distribuição percentual dos candidatos, por área escolhida, segundo variáveis demográficas, socioeconômicas e culturais.

\begin{tabular}{|c|c|c|c|c|c|}
\hline \multirow[b]{2}{*}{ Variáveis } & \multirow[b]{2}{*}{$\begin{array}{l}\text { Percentual de } \\
\text { candidatos }\end{array}$} & \multicolumn{3}{|c|}{ Área escolhida } & \multirow[b]{2}{*}{ Total } \\
\hline & & Engenharias & $\begin{array}{l}\text { Ciências } \\
\text { exatas }\end{array}$ & $\begin{array}{c}\text { Ciências } \\
\text { humanas/ } \\
\text { biológicas }\end{array}$ & \\
\hline \multicolumn{6}{|l|}{ Sexo } \\
\hline Masculino & 37,5 & 28,9 & 14,3 & 56,8 & 100 \\
\hline Feminino & 62,5 & 11,8 & 6,5 & 81,8 & 100 \\
\hline \multicolumn{6}{|l|}{ Idade } \\
\hline De 15 a 18 anos & 27,3 & 23,8 & 9,1 & 67,1 & 100 \\
\hline De 19 a 20 anos & 47,1 & 18,8 & 9,3 & 71,9 & 100 \\
\hline 21 anos ou mais & 25,6 & 10,9 & 9,9 & 79,2 & 100 \\
\hline \multicolumn{6}{|l|}{ Cidade } \\
\hline Rio de Janeiro & 35,4 & 19,8 & 10,2 & 70,0 & 100 \\
\hline Niterói & 12,7 & 19,0 & 11,2 & 69,8 & 100 \\
\hline São Gonçalo & 12,7 & 14,5 & 14,1 & 71,3 & 100 \\
\hline Outra & 39,2 & 17,6 & 6,5 & 75,9 & 100 \\
\hline \multicolumn{6}{|l|}{ Ensino Médio } \\
\hline Público & 38,1 & 16,1 & 11,1 & 72,8 & 100 \\
\hline Particular & 61,9 & 19,5 & 8,3 & 72,2 & 100 \\
\hline \multicolumn{6}{|l|}{ Ano de conclusão } \\
\hline 2010 & 48,0 & 21,9 & 9,5 & 68,6 & 100 \\
\hline 2009 & 23,4 & 18,4 & 9,2 & 72,4 & 100 \\
\hline Antes & 28,6 & 11,8 & 9,3 & 78,9 & 100 \\
\hline \multicolumn{6}{|l|}{$\begin{array}{l}\text { Frequentou outro } \\
\text { curso }\end{array}$} \\
\hline Não frequentou & 43,4 & 20,7 & 10,5 & 68,8 & 100 \\
\hline Frequentou & 56,6 & 16,2 & 8,5 & 75,2 & 100 \\
\hline \multicolumn{6}{|l|}{$\begin{array}{l}\text { Prestou vestibular } \\
\text { antes }\end{array}$} \\
\hline Não prestou & 33,9 & 19,8 & 9,9 & 70,4 & 100 \\
\hline $1 \mathrm{vez}$ & 39,4 & 19,0 & 9,8 & 71,2 & 100 \\
\hline 2 vezes ou mais & 26,8 & 15,0 & 8,1 & 76,9 & 100 \\
\hline \multicolumn{6}{|l|}{ Motivo da escolha } \\
\hline $\begin{array}{l}\text { Adequação as aptidões } \\
\text { pessoais }\end{array}$ & 62,9 & 15,2 & 8,6 & 76,2 & 100 \\
\hline Motivos Financeiros & 15,3 & 32,4 & 11,7 & 55,9 & 100 \\
\hline Outro & 21,8 & 16,9 & 10,0 & 73,1 & 100 \\
\hline \multicolumn{6}{|l|}{ Cor } \\
\hline Branca & 62,4 & 19,0 & 9,1 & 71,9 & 100 \\
\hline Não branca & 37,6 & 16,7 & 9,9 & 73,3 & 100 \\
\hline \multicolumn{6}{|l|}{ Instrução do pai } \\
\hline $\begin{array}{l}\text { No máximo } \\
\text { Fundamental }\end{array}$ & 25,2 & 13,6 & 11,0 & 75,4 & 100 \\
\hline Médio & 34,6 & 18,4 & 10,1 & 71,5 & 100 \\
\hline Superior ou pós & 40,2 & 20,8 & 7,8 & 71,4 & 100 \\
\hline \multicolumn{6}{|l|}{ Instrução da mãe } \\
\hline $\begin{array}{l}\text { No máximo } \\
\text { Fundamental }\end{array}$ & 21,8 & 12,7 & 11,0 & 76,3 & 100 \\
\hline Médio & 34,8 & 19,3 & 10,5 & 70,1 & 100 \\
\hline Superior ou pós & 43,4 & 20,0 & 7,7 & 72,3 & 100 \\
\hline
\end{tabular}


Renda mensal familiar

Até 3 salários mínimos

Mais de 3 a 10 salários

mínimos

Mais de 10 salários

mínimos

39,0

36,3

24,7

85,9

14,1

85,7

14,3

Outro

Domínio de língua

estrangeira

Sim

Não
13,7

20,3

22,2

18,9

13,6

19,2

11,8

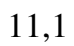

8,7

7,7

8,6

14,1

9,7

7,5
75,2

71,0

70,2

72,5

72,3

71,1

80,7

8,7

10,7
71,4

74,5
100

100

100

100

100

100

100
$\mathrm{Na}$ Tabela 1, verifica-se que $37,5 \%$ dos candidatos são do sexo masculino e $62,5 \%$ são do sexo feminino. Entre os candidatos do sexo masculino, se observa maior proporção de indivíduos que escolheram as áreas de Engenharias (Homens: 28,9\%; Mulheres: 11,8\%).

Com relação a idade dos candidatos, verifica-se que quase a metade dos candidatos $(47,1 \%)$ estão na faixa etária de 19 a 20 anos. Entre os candidatos com 15 a 18 anos de idade, observa-se maior proporção daqueles que escolheram as áreas de engenharia $(23,8 \%)$, enquanto que nos grupos etários de "19 a 20 anos" e "21 anos ou mais", a proporção de indivíduos que escolheram as áreas de engenharia é de $18,8 \%$ e $10,9 \%$, respectivamente.

Quanto à cidade de residência, observa-se que $35,4 \%$ dos candidatos residem na cidade do Rio de Janeiro, enquanto que $25,4 \%$ dos candidatos residem nas cidades de Niterói ou São Gonçalo (12,7\% em cada cidade). Os demais candidatos $(39,2 \%)$ são provenientes de outras cidades. A proporção de indivíduos que escolheram as áreas de engenharia é maior nas cidades de Niterói $(19,0 \%)$ e Rio de Janeiro $(19,8 \%)$, e menor na cidade de São Gonçalo (14,5\%).

A maioria dos candidatos é proveniente de escolas particulares $(61,9 \%)$. Comparando a proveniência do ensino médio, observou-se maior percentual de candidatos provenientes do ensino particular escolhendo as áreas de engenharias (particular: 19,5\%; público: $16,1 \%)$. Quase metade dos candidatos $(48,0 \%)$ estava por encerrar o ensino médio no mesmo ano do vestibular (ano de 2010) e a maioria (56,6\%) dos candidatos frequentou outros cursos em sua preparação para o vestibular. Ao comparar os anos de conclusão, observase que em 2010, há maior percentual de candidatos que escolheram as áreas de engenharia $(21,9 \%)$.

Quanto a informação do candidato já ter prestado vestibular anteriormente, $39,4 \%$ responderam que já tinham prestado vestibular uma vez, enquanto $26,8 \%$ dos candidatos informaram que já tinham prestado ao menos duas vezes. Os demais candidatos $(33,9 \%)$ estavam prestando vestibular pela primeira vez.

Com relação a motivação na escolha do curso, 62,9\% dos candidatos levaram em consideração a adequação do curso às suas aptidões pessoais e 15,3\% foram motivados principalmente por fatores financeiros, como maior oferta no mercado de trabalho ou recompensa financeira mais promissora. Dentre os candidatos motivados por questões financeiras, $32,4 \%$ escolheram os cursos das áreas de engenharia, proporção consideravelmente maior que a observada entre os candidatos que alegaram outros motivos para sua escolha pelas áreas de 
engenharia (aptidão pessoal: 15,2\%; outro motivo: $16,9 \%$ ).

A maioria dos candidatos $(62,4 \%)$ é de cor branca; cerca de $40 \%$ dos candidatos são filhos de pais ou mães com ensino superior ou pós-graduação, sendo nestes grupos, observado maior percentual de candidatos que escolheram as áreas de engenharia (pai: 20,8\%; mãe: $20,0 \%$ ).

Com relação as características econômicas, observa-se maior proporção de candidatos que não estão trabalhando $(85,9 \%)$ e que possuem renda mensal familiar de até 3 salários mínimos $(39,0 \%)$. Observa-se ainda que há maior proporção de candidatos escolhendo as áreas de engenharia, na faixa de renda mais elevada. Além disso, observou-se maior proporção de candidatos escolhendo as áreas de engenharia no grupo dos candidatos que não trabalham (não trabalha: 18,9\%; trabalha:13,6\%).

Do total de candidatos, $85,7 \%$ moram na casa dos pais. Verifica-se maior proporção pela escolha das áreas de engenharia, entre os candidatos que moram com os pais, comparativamente aos que não moram com os pais. Quanto a língua estrangeira, $66,9 \%$ dos candidatos declararam ter domínio de alguma língua estrangeira, dos quais $19,9 \%$ fizeram a opção pelas áreas engenharias.

A Tabela 2 fornece os resultados dos testes de significância geral dos parâmetros do modelo logístico multinomial explicativo da área escolhida pelos candidatos, considerando todas as variáveis de estudo.
Tabela 2: Resultados dos testes de significância geral para os parâmetros do modelo logístico multinomial explicativo da área escolhida pelos candidatos, considerando todas as variáveis de estudo.

\begin{tabular}{lr}
\hline Variável & p-valor \\
\hline Sexo & $<0,001$ \\
Idade & $<0,001$ \\
Cidade & $<0,001$ \\
Ensino Médio & $<0,001$ \\
Ano de conclusão & 0,001 \\
Frequentou outro curso & $<0,001$ \\
Prestou vestibular antes & 0,310 \\
Motivo da escolha & $<0,001$ \\
Cor & 0,218 \\
Instrução do pai & 0,018 \\
Instrução da mãe & $<0,001$ \\
Renda mensal familiar & $<0,001$ \\
Trabalho & $<0,001$ \\
Onde mora & $<0,001$ \\
Domínio de língua estrangeira & 0,007 \\
\hline
\end{tabular}

Apenas as variáveis "prestou vestibular antes" e "cor" não apresentaram associação estatisticamente significante com a escolha da área de estudo considerando o nível de significância de $5 \%$ (p-valor > 5\%) (Tabela 2).

A Tabela 3 fornece os resultados do ajuste do modelo considerando apenas as variáveis de estudo estatisticamente relacionadas com a chance do candidato escolher as áreas de Engenharia, relativamente as áreas de Ciências Exatas e as áreas de Ciências Humanas/Biológicas. 
Tabela 3: Resultados do ajuste do modelo logístico multinomial (modelo selecionado) explicativo da área escolhida pelos candidatos.

\begin{tabular}{|c|c|c|c|c|c|c|}
\hline \multirow[t]{2}{*}{ Variáveis } & \multicolumn{3}{|c|}{$\begin{array}{c}\text { Engenharias vs Ciências } \\
\text { exatas }\end{array}$} & \multicolumn{3}{|c|}{$\begin{array}{l}\text { Engenharias vs Ciências } \\
\text { humanas/biológicas }\end{array}$} \\
\hline & Estimativa & OR & P-valor & Estimativa & OR & P-valor \\
\hline Intercepto & 0,624 & - & $<0,001$ & $-3,041$ & - & $<0,001$ \\
\hline \multicolumn{7}{|l|}{ Sexo } \\
\hline Masculino & 0,049 & 1,051 & 0,319 & 1,248 & 3,485 & $<0,001$ \\
\hline Feminino & 0 & 1 & - & 0 & 1 & - \\
\hline \multicolumn{7}{|l|}{ Idade } \\
\hline Menos de 19 anos & 0,396 & 1,485 & $<0,001$ & 0,737 & 2,090 & $<0,001$ \\
\hline De 19 a 20 anos & 0,225 & 1,253 & 0,012 & 0,462 & 1,588 & $<0,001$ \\
\hline Mais de 20 anos & 0 & 1 & - & 0 & 1 & - \\
\hline \multicolumn{7}{|l|}{ Cidade } \\
\hline Rio de Janeiro & $-0,472$ & 0,624 & $<0,001$ & 0,054 & 1,055 & 0,165 \\
\hline Niterói & $-0,504$ & 0,604 & $<0,001$ & 0,049 & 1,050 & 0,354 \\
\hline São Gonçalo & $-0,670$ & 0,512 & $<0,001$ & $-0,046$ & 0,955 & 0,426 \\
\hline Outra & 0 & 1 & - & 0 & 1 & - \\
\hline \multicolumn{7}{|l|}{ Ensino Médio } \\
\hline Público & 0,046 & 1,047 & 0,430 & 0,174 & 1,191 & $<0,001$ \\
\hline Particular & 0 & 1 & - & 0 & 1 & - \\
\hline \multicolumn{7}{|l|}{ Ano de conclusão } \\
\hline 2010 & 0,125 & 1,133 & 0,160 & 0,236 & 1,267 & $<0,001$ \\
\hline 2009 & 0,108 & 1,114 & 0,227 & 0,252 & 1,287 & $<0,001$ \\
\hline Antes & 0 & 1 & - & 0 & 1 & - \\
\hline \multicolumn{7}{|l|}{ Frequentou outro curso } \\
\hline Não frequentou & $-0,062$ & 0,940 & 0,232 & 0,099 & 1,104 & 0,006 \\
\hline Frequentou & 0 & 1 & - & 0 & 1 & - \\
\hline \multicolumn{7}{|l|}{ Motivo da escolha } \\
\hline $\begin{array}{l}\text { Adequação as aptidões } \\
\text { pessoais }\end{array}$ & $-0,069$ & 0,933 & 0,250 & $-0,224$ & 0,800 & $<0,001$ \\
\hline Motivos financeiros & 0,476 & 1,610 & $<0,001$ & 0,841 & 2,320 & $<0,001$ \\
\hline Outro & 0 & 1 & - & 0 & 1 & - \\
\hline \multicolumn{7}{|l|}{ Instrução do pai } \\
\hline No máximo Fundamental & $-0,217$ & 0,805 & 0,009 & $-0,036$ & 0,964 & 0,525 \\
\hline Médio & $-0,131$ & 0,877 & 0,040 & 0,033 & 1,033 & 0,431 \\
\hline Superior ou pós & 0 & 1 & - & 0 & 1 & - \\
\hline \multicolumn{7}{|l|}{ Instrução da mãe } \\
\hline No máximo Fundamental & $-0,182$ & 0,833 & 0,030 & $-0,030$ & 0,970 & 0,606 \\
\hline Médio & $-0,045$ & 0,956 & 0,463 & 0,200 & 1,221 & $<0,001$ \\
\hline Superior ou pós & 0 & 1 & - & 0 & 1 & - \\
\hline \multicolumn{7}{|l|}{ Renda mensal familiar } \\
\hline Até 3s.m & $-0,508$ & 0,602 & $<0,001$ & $-0,275$ & 0,759 & $<0,001$ \\
\hline Mais de 3 a 10s.m & $-0,110$ & 0,895 & 0,106 & 0,027 & 1,028 & 0,524 \\
\hline Mais de 10 s.m & 0 & 1 & - & 0 & 1 & - \\
\hline \multicolumn{7}{|l|}{ Trabalho } \\
\hline Não trabalha & 0,359 & 1,432 & $<0,001$ & $-0,046$ & 0,955 & 0,431 \\
\hline Trabalha & 0 & 1 & - & 0 & 1 & - \\
\hline
\end{tabular}




\begin{tabular}{lcccccc}
\hline Onde mora & & & & & & \\
Na casa dos pais & $-0,063$ & 0,939 & 0,437 & 0,358 & 1,430 & $<0,001$ \\
$\begin{array}{l}\text { Outro } \\
\text { Domínio de língua } \\
\text { estrangeira }\end{array}$ & 0 & 1 & - & 0 & 1 & - \\
Sim & 0,162 & 1,176 & 0,004 & 0,097 & 1,102 & 0,016 \\
Não & 0 & 1 & - & 0 & 1 & - \\
\hline
\end{tabular}

Os candidatos do sexo masculino têm uma chance de escolher as áreas de Engenharia relativamente as áreas de Ciências Humanas/Biológicas 3,485 vezes maior do que candidatos do sexo feminino (Tabela 3). O fato do sexo dos candidatos estar associado substancialmente com a escolha do curso, já havia sido observado por Bahia \& Laudares (2011), destacando que a engenharia carrega a marca da masculinidade mais enraizada, sendo ainda vista como uma profissão para homens.

Com relação a variável "idade", observou-se que a chance dos candidatos escolherem as áreas de Engenharia diminui à medida que aumenta a faixa etária, tanto em comparação aos candidatos que escolheram as demais áreas de Ciências exatas quanto aos candidatos que escolheram as áreas de Ciências humanas/biológicas. Esta tendência de quanto menor a faixa etária, maior a chance de escolher as áreas de Engenharia, também foi verificado no estudo de Souza et al. (2012), que objetivou avaliar a escolha profissional pelos cursos de engenharia da Universidade do Estado de Santa Catarina (UDESC), mas numa análise voltada especificamente para estudantes do sexo feminino.

Com relação à cidade de residência, a chance dos candidatos escolherem as áreas de Engenharia comparada com as demais áreas de Ciências Exatas, foi menor, sobretudo na cidade de São Gonçalo, relativamente aos candidatos residentes em outras cidades.

Quanto a proveniência do ensino médio, verificou-se que os candidatos que estudaram no ensino público possuem uma chance $19,1 \%$ maior de optar pelas áreas de Engenharia, em comparação às áreas de Ciências humanas/biológicas
$(\mathrm{RC}=1,191 ; \quad \mathrm{p}$-valor $<0,001) . \quad \mathrm{Se}$ basearmos no achado de Melo-Silva et al. (2008), de que existe maior maturidade entre os alunos provenientes das escolas do ensino público, pode-se concluir que estes alunos estão mais conscientes de sua escolha pelas áreas de Engenharia.

Os candidatos que concluíram o ensino médio em 2009 ou 2010 possuem uma chance aproximadamente $28 \%$ maior de escolherem as áreas de Engenharia vs Ciências humanas/biológicas, em relação aos candidatos que concluíram o ensino médio antes de 2009.

Os candidatos que não frequentaram curso pré-vestibular ou curso de matérias isoladas apresentaram uma chance $10,4 \%$ maior de escolherem as áreas de Engenharia vs Ciências humanas/biológicas $\quad(\mathrm{RC}=1,104 ; \quad \mathrm{p}$ valor $=0,006), \quad$ relativamente aos candidatos que frequentaram pelo menos um desses cursos.

A chance de escolher as áreas de Engenharia comparada com as áreas de Ciências exatas foi $61,0 \%$ maior para os candidatos que declararam motivação financeira em relação aos candidatos que reportaram outro motivo $(\mathrm{RC}=1,610$; $\mathrm{p}$ valor $<0,001)$. Já a chance de escolher as áreas de Engenharia comparada com as áreas de Ciências humanas/biológicas foi 2,320 vezes maior para os que declararam motivação financeira $(\mathrm{RC}=2,320 ; \mathrm{p}$ valor $<0,001)$. No estudo de Hirt \& Raitz(2009), também foi verificado que o salário foi o fator mais reportado pelos jovens para a escolha da área profissional, onde $27 \%$ dos jovens relataram que o salário interfere nesta escolha. A aptidão, portanto, parece não ser um fator prioritário para a escolha das áreas de Engenharia, o que é preocupante, pois como foi verificado no estudo de Bardagi (2006), a identificação pessoal é um fator relacionado à satisfação com a 
escolha da área profissional. Inclusive, no presente estudo, verificou-se que a chance de optar pelas áreas de Engenharias, relativamente as áreas de Ciências humanas/biológicas, é menor para candidatos que apontaram a aptidão como fator de escolha pela área profissional ( $\mathrm{RC}=0,800$; $\mathrm{p}$-valor $<0,001)$.

Com relação ao "nível de instrução do pai", observou-se que a chance de escolher Engenharias vs Ciências exatas é 14,0\% maior para os candidatos cujos pais tem ensino superior ou pós-graduação, relativamente aos candidatos filhos de pais com ensino médio $\quad(\mathrm{RC}=1 / 0,877=1,140 ; \quad \mathrm{p}$ valor $=0,040)$. E essa chance é $24,2 \%$ maior em relação aos candidatos filhos de pais com no máximo ensino fundamental ( $\mathrm{RC}=1 / 0,805=1,242 ; \mathrm{p}$-valor=0,009). A variável "instrução do pai" não teve efeito estatisticamente significante na chance do candidato escolher Engenharias vs Ciências humanas/biológicas (Tabela 3).

Com relação ao "nível de instrução da mãe", observou-se que a chance de escolher Engenharias vs Ciências exatas é 20,0\% maior para os candidatos cujas mães tem ensino superior ou pós-graduação, relativamente aos candidatos filhos de mães com no máximo ensino fundamental ( $\mathrm{RC}=1 / 0,833=1,200 ; \mathrm{p}$-valor=0,030). A chance de escolher as áreas de Engenharias vs Ciências humanas/biológicas é $22,1 \%$ maior $(\mathrm{RC}=1,221 ; \quad \mathrm{p}$-valor $<0,001)$ para os candidatos cujas mães tem ensino médio, em relação aos candidatos filhos de mães com ensino superior ou pós-graduação. Segundo Noronha \& Ottati (2010), pais e mães mais escolarizados possuem maior influência na escolha profissional dos filhos pelas áreas de Engenharia, que caracterizam áreas profissionais reconhecidamente tradicionais. Também foi observado neste trabalho que candidatos filhos de pais com formação superior ou pós-graduação possuem interesses pelas áreas de Engenharia, relativamente a outras áreas de Ciências Exatas.
A chance dos candidatos com renda mensal familiar de até 3 salários mínimos (faixa mais baixa), relativamente àqueles com renda mensal familiar com mais de 10 salários mínimos (faixa mais alta), escolherem as áreas de Engenharias é aproximadamente $40 \%$ menor em comparação com a área de Ciências exatas $\quad(\mathrm{RC}=0,602, \quad \mathrm{p}$ valor $<0,001)$ e $24,1 \%$ menor comparativamente a área de Ciências humanas/biológicas $\quad(\mathrm{RC}=0,759, \quad \mathrm{p}$ valor $<0,001)$. Estes resultados podem ser explicados pelo fato de que cursos de maior prestígio social e maior remuneração, como as Engenharias, costumam atrair jovens de faixas de renda mais alta (Borges \& Carnielli, 2005).

Os candidatos que não trabalham possuem uma chance $43,2 \%$ maior de escolher Engenharias vs Ciências exatas $(\mathrm{RC}=1,432 ; \mathrm{p}$-valor $<0,001)$. Sendo assim, o fato de não trabalhar pode estar associado ao desejo do candidato pelo curso e ao conhecimento de que os primeiros anos dos cursos de Engenharia são mais difíceis, dada a grande variedade de disciplinas de Cálculo, Física e Química.

Os candidatos que moram com os pais têm uma chance 43,0\% maior de escolher Engenharias vs Ciências humanas/biológicas $\quad(\mathrm{RC}=1,430 ; \quad \mathrm{p}$ valor $<0,001$ ).

A chance do candidato escolher as áreas de Engenharias relativamente as de Ciências Exatas é 17,6\% maior para os candidatos com domínio de alguma língua estrangeira $(\mathrm{RC}=1,176$; $\mathrm{p}$ valor=0,004). Já a chance de escolher as áreas de Engenharias relativamente as de Ciências humanas/biológicas é 10,2\% maior para os candidatos com domínio de língua estrangeira $\quad(\mathrm{RC}=1,102 ; \quad \mathrm{p}$ valor $=0,016)$. Atualmente, o mercado de trabalho em qualquer área tem exigido o conhecimento de língua estrangeira, especialmente o inglês. Para a área de Engenharias, outras línguas também são importantes (Zago, 2006).

No que se refere a qualidade do ajuste, observou-se que o modelo selecionado classificou corretamente $72,8 \%$ dos candidatos. Além disso, 
através do teste de significância baseado no Índice de Kappa, verificou-se que existe concordância significativa entre as categorias (áreas de estudo) observadas e preditas do modelo ( $\mathrm{p}$-valor $<0,001$ ).

Quanto a limitação do estudo, pode-se citar a não obrigatoriedade do preenchimento do questionário sociocultural na fase de inscrição dos candidatos ao Vestibular da UFF 2011. Com isso, a base de dados foi reduzida, pela exclusão dos candidatos que optaram por não responder $\mathrm{o}$ questionário $\mathrm{e}$ daqueles que não responderam questões relativas as variáveis utilizadas no estudo.

\section{CONCLUSÕES}

O presente trabalho mostrou que existe associação entre múltiplos fatores e a escolha da área profissional, em particular a áreas de Engenharia. As variáveis sexo e o tipo de motivação tiveram os maiores efeitos sobre a chance de escolher as áreas de Engenharia, sendo esta chance maior para os candidatos do sexo masculino e para aqueles que alegaram motivações de cunho financeiro para sua escolha. As associações entre essas variáveis e a chance de escolha pelas áreas de Engenharia foram mais fortes quando comparados com as áreas de Ciências Humanas/Biológicas do que quando comparados com as áreas de Ciências Exatas. Observou-se ainda maior chance de escolher as áreas de Engenharia, em comparação com as outras duas áreas profissionais, para aqueles candidatos: com faixa etária de 15 a 19 anos, com maior renda mensal familiar (mais de $10 \mathrm{~s} . \mathrm{m} v s$ até $3 \mathrm{~s} . \mathrm{m}$ ) e com domínio de língua estrangeira.

Além disso, conclui-se que candidatos que moram em outra cidade (que não Rio de Janeiro, Niterói e São Gonçalo), que tem pais ou mães com ensino superior ou pós-graduação e que não trabalham apresentam maior chance de escolher as áreas de Engenharia, quando comparadas com as demais áreas de Ciências Exatas. Quando comparadas com as áreas de Ciências Humanas/Biológicas, observou-se maior chance de escolha pelas áreas de
Engenharia para candidatos que: moram com os pais, são provenientes de escola pública, concluíram o ensino médio em 2010 ou em 2009, não frequentaram cursos complementares para a preparação para o vestibular e que possuem mães com nível médio.

Diante da complexidade acerca da escolha profissional, ressalta-se a importância da orientação profissional, desenvolvida por equipe multiprofissional qualificada em escolas do ensino médio, a fim de identificar as características peculiares dos alunos, auxiliando-os em suas escolhas profissionais.

\section{REFERÊNCIAS}

BAHIA, M.M; LAUDARES, J.B., 2011. A participação da mulher em áreas específicas $d a$ engenharia. XXXIX Congresso Brasileiro de Educação em Engenharia. Blumenau - SC. Disponível em: $<$ http://www.abenge.org.br/CobengeA nteriores/2011/sessoestec/art1619.pdf>

BARDAGI, M.; LASSANCE, M. C. P.; PARADISO, Â. C.; MENEZES, I. A., 2006. Escolha profissional e inserção no mercado de trabalho: percepções de estudantes formandos. Psicol. Esc. Educ. (online). 10 (1), 69-82.

BIANCHINI, B.M.; FRAGA, D.P.F.; CURVELLO, J.C.R; MADEIRA, S.D.S.; CAMPOS, O.C.; SANTIAGO, M.E.V.; MENDES, R.S., 2011. A comunicação como fator preponderante para o novo perfil do engenheiro. In: VII Seminário Fluminense de Engenharia, Niterói-RJ. Disponível em: $<$ http://www.engenharia.uff.br/images/sto ries/noticias/anaisviiseminriofluminensed eengenhariauff2011.pdf $>$.

BORGES, J. L. G. \& CARNIELLI, B. L. 2005. Educação e estratificação social no acesso à universidade pública. Cadernos de Pesquisa. São Paulo. 35 (124), 113-139.

BRASIL. Lei n. 9.394, de 20 de dezembro de 1996. Estabelece as 
Diretrizes e Bases da Educação Nacional. Disponível em: $<$ http://www.planalto.gov.br/CCIVIL_03/ leis/L9394.htm>.

CARDOSO, V. L. S.; MAINIER, F.B.; SILVA, R. R. C. M..2011. Engenharia química e mercado de trabalho: percepções discentes. In: VII Seminário Fluminense de Engenharia. Niterói-RJ. Disponível em: <http://www.engenharia.uff.br/images/sto ries/noticias/anaisviiseminriofluminensed eengenhariauff2011.pdf>.

CASTANHO, D. M.; FREITAS, S. N. 2006. Inclusão e prática docente no ensino superior. Centro de Educação. Revista Brasileira de Educação Especial, Santa Maria, 27, 93-99.

CONFEA. Propostas para o Desenvolvimento Sustentável Brasileiro. Brasília, 2010. Disponível em: $<$ http://www.confea.org.br/media/propost as_des.pdf $>$.

FILHO, N. G. S.; SANTANA, J. G. L.; SILVA, L. R. B.2011. A responsabilidade social na vida de um engenheiro. In: XXXIX Congresso Brasileiro de Educação em Engenharia. Blumenau-SC. Disponível em: $<$ http://www.abenge.org.br/CobengeAnte riores/2011/sessoestec/art2095.pdf>.

GUIMARÃES, N. N. C. C.; MAINIER, F. B.; SILVA, R. R. C. M. 2011. Análise de fatores que contribuem para a redução no índice de evasão. In: VII Seminário Fluminense de Engenharia. Niterói-RJ. Disponível em: $<$ http://www.engenharia.uff.br/images/sto ries/noticias/anaisviiseminriofluminensed eengenhariauff2011.pdf>.

HIRT, L.U.; RAITZ, T.R. 2009. Jovens do ensino médio em busca de orientação profissional: uma proposta com grupos focais. XV Encontro Nacional da Abrapso, Maceió-AL.

KUTNER,M.H.; NACHTSHEIM， C.J.; NETER, J. 2004. Applied Linear
Regression Models. 4thed. New York: McGraw-Hill/Irwin.

LONGO, W. P. R. 1996. Reengenharia do ensino de engenharia: uma necessidade. Revista Segurança \& Desenvolvimento, 220, 13-16.

MARÔCO, J. 2010.Análise Estatística: Com o PASW Statistics (ex-SPSS). Pêro Pinheiro: ReportNumber.

MELO-SILVA, L.; JUNQUEIRA, M. L.; FARIA, L. 2008.Avaliação da maturidade para a escolha profissional: estudo comparativo com alunos do ensino médio provindos de escolas da rede pública e da rede particular de ensino no Brasil. Conferência Internacional de Avaliação Psicológica: Formas e Contextos". Portugal.

NORONHA, A. P. P.; OTTATI, F. 2010. Interesses profissionais de jovens $e$ escolaridade dos pais. Rev. bras. orientac. prof, São Paulo, 11(1), 37-47.

SACAVINO, S. 2006. Direito humano à educação no Brasil: uma conquista para todos/as. In: SILVEIRA, R. M. G. (Org.). Educação em Direitos Humanos: fundamentos teórico-metodológicos. João Pessoa: Editora Universitária, 457-467.

SILVA, L.P.; CECÍLIO, S. 2007. A Mudança no Modelo de Ensino e de Formação na Engenharia. Educação em Revista, Belo Horizonte-MG, 45, 61-80.

SOUZA, M.E.; BENEVENUTTI, V.; BITTENCOURT, E. 2012.A escolha, o curso e a expectativa profissional das mulheres estudantes dos cursos de engenharia da universidade do estado de Santa Catarina - Udesc. XL Congresso Brasileiro de Educação em Engenharia. Belém - PA.

SPARTA, M.; GOMES, W. G. 2005. Importância atribuída ao ingresso na educação superior por alunos do ensino médio. Revista brasileira de Orientação Profissional, São Paulo, 6(2), 45-53. 
ZAGO, N. 2006. Do acesso à permanência no ensino superior: percursos de estudantes universitários de camadas populares. Revista Brasileira de Educação, 11(32): 226-370. 